\title{
Weierstrass integrability for a class of differential systems
}

Jaume Llibre, Claudia Valls

To cite this article: Jaume Llibre, Claudia Valls (2014) Weierstrass integrability for a class of differential systems, Journal of Nonlinear Mathematical Physics 21:2, 294-307, DOI: https://doi.org/10.1080/14029251.2014.915165

To link to this article: https://doi.org/10.1080/14029251.2014.915165

Published online: 04 January 2021 


\title{
Weierstrass integrability for a class of differential systems
}

\author{
Jaume Llibre \\ Departament de Matemàtiques, Universitat Autònoma de Barcelona, \\ 08193 Bellaterra, Barcelona, Catalonia, Spain \\ jllibre@mat.uab.cat \\ Claudia Valls* \\ Departamento de Matemática, Instituto Superior Técnico, Universidade Técnica de Lisboa, \\ Av. Rovisco Pais 1049-001, Lisboa, Portugal \\ cvalls@math.ist.utl.pt
}

Received 28 January 2014

Accepted 19 March 2014

We characterize the differential equations of the form

$$
x^{\prime}=y, \quad y^{\prime}=a_{n}(x) y^{n}+a_{n-1}(x) y^{n-1}+\cdots+a_{1}(x) y+a_{0}(x), \quad n \geq 2, \quad a_{n}(0) \neq 0,
$$

where $a_{j}(x)$ are meromorphic functions in the variable $x$ for $j=0, \ldots, n$ that admits either a Weierstrass first integral or a Weierstrass inverse integrating factor.

Keywords: Weierstrass first integrals; Weierstrass inverse integrating factor; complex differential systems.

2000 Mathematics Subject Classification: 34C05, 34A34, 34C14

\section{Introduction and statement of the main results}

Let $x$ and $y$ be complex variables. In this paper we study the differential equations of the form

$$
\begin{aligned}
& x^{\prime}=y=P(x, y), \\
& y^{\prime}=a_{n}(x) y^{n}+a_{n-1}(x) y^{n-1}+\cdots+a_{1}(x) y+a_{0}(x)=Q(x, y),
\end{aligned}
$$

where $n \geq 2, a_{n}(0) \neq 0$, the functions $a_{j}(x)$ are meromorphic functions in the variable $x$ for $j=$ $0, \ldots, n$, and the prime indicates derivative with respect to the time $t$, real or complex.

The goal of this paper is to analyze the integrability of the differential systems (1.1) restricted to a special kind of first integrals. For such systems the notion of integrability is based on the existence of a first integral, and we shall characterize when the differential system (1.1) has a Weierstrass first integral or a Weierstrass inverse integrating factor. More precisely, guided by the fact that system (1.1) is polynomial in the variable $y$, we study the first integrals and the inverse integrating factors that are polynomials in the variable $y$ and are an analytic function in the variable $x$, i.e. we study the so called Weierstrass integrability of system (1.1).

\footnotetext{
*The second author is supported by Portuguese National Funds through FCT - Fundação para a Ciência e a Tecnologia within the project PTDC/MAT/117106/2010 and by CAMGSD.
} 
As usual $\mathbb{C}[[x]]$ is the ring of formal power series in the variable $x$ with coefficients in $\mathbb{C}$, and $\mathbb{C}[y]$ is the ring of polynomials in the variable $y$ with coefficients in $\mathbb{C}$. A polynomial of the form

$$
\sum_{i=0}^{n} w_{i}(x) y^{i} \in \mathbb{C}[[x]][y]
$$

is called a formal Weierstrass polynomial in the variable $y$ of degree $n$ if and only if $w_{n}(x)=1$ and $w_{i}(0)=0$ for $i<n$. A formal polynomial whose coefficients are convergent is called Weierstrass polynomial, see [1]. In other words, a Weierstrass polynomial first integral is of the form

$$
H=y^{s}+H_{s-1}(x) y^{s-1}+\cdots+H_{1}(x) y+H_{0}(x)=\sum_{i=0}^{s} H_{i}(x) y^{i}
$$

(with $H_{s}(x)=1$ ).

We recall that an analytic first integral $H: U \rightarrow \mathbb{C}$ of system (1.1) where $U$ is an open subset of $\mathbb{C}^{2}$ is a non-locally constant analytic function such that it is constant on the solution of system (1.1) contained in $U$.

In what follows we prefer to work with an inverse integrating factor $V=V(x, y)$ than with an integrating factor $R=R(x, y)=1 / V(x, y)$, because both work well for finding first integrals, but $V$ has better properties than $R$, for instance in the region where $V$ is defined if there are limit cycles they are contained in $V=0$, see more raisons in the paper [6].

An inverse integrating factor for the differential system (1.1) is a function $V$ defined in an open subset of the domain of definition of the differential system that satisfies

$$
\frac{\partial(P / V)}{\partial x}+\frac{\partial(Q / V)}{\partial y}=0
$$

or equivalently

$$
\frac{\partial V}{\partial x} P+\frac{\partial V}{\partial y} Q=\left(\frac{\partial P}{\partial x}+\frac{\partial Q}{\partial y}\right) V
$$

i.e. if $V$ is an inverse integrating factor of our differential system (1.1) it must satisfy

$$
\begin{aligned}
& \frac{\partial V}{\partial x} y+\frac{\partial V}{\partial y}\left(a_{n}(x) y^{n}+a_{n-1}(x) y^{n-1}+\cdots+a_{1}(x) y+a_{0}(x)\right) \\
& =\left(n a_{n}(x) y^{n-1}+(n-1) a_{n-1}(x) y^{n-2}+\cdots+a_{1}(x)\right) V .
\end{aligned}
$$

Therefore if $V$ is an inverse integrating factor of our differential system (1.1), then it is known that there exists a first integral $H$ of the Hamiltonian system

$$
\begin{aligned}
& \dot{x}=\frac{y}{V}=\frac{\partial H}{\partial y}, \\
& \dot{y}=\frac{a_{n}(x) y^{n}+a_{n-1}(x) y^{n-1}+\cdots+a_{1}(x) y+a_{0}(x)}{V}=-\frac{\partial H}{\partial x} .
\end{aligned}
$$

Now from the first equation we obtain

$$
H=\int \frac{P}{V} d y+f(x)
$$


and substituting this $H$ in the second equation we determine the function $f(x)$, and consequently the first integral $H$. So for studying the integrability (i.e. the existence of a first integral) of the differential system (1.1) it is sufficient to determine an inverse integrating factor of system (1.1).

We say that a differential system (1.1) is Weierstrass integrable if it admits either a first integral or an inverse integrating factor which is a Weierstrass polynomial. In [6] this definition is given in a more general context.

The main objective of this paper is to provide the differential systems (1.1) that have Weierstrass first integrals, or Weierstrass integrating factors. More precisely: How to determine the functions $a_{j}(x)$, for $j=1, \ldots, n$ and $n \geq 2$ for which the differential systems (1.1) are Weierstrass integrable?

Unfortunately the differential systems (1.1) have no Weierstrass first integrals as the following results shows.

Proposition 1.1. The differential systems (1.1) have no Weierstrass first integrals.

Proposition 1.1 is proved in section 2.

In view of Proposition 1.1 we will concentrate on investigating the inverse integrating factors of the form

$$
V=y^{s}+V_{s-1}(x) y^{s-1}+\cdots+V_{1}(x) y+V_{0}(x)=\sum_{i=0}^{s} V_{i}(x) y^{i}
$$

with $V_{s}(x)=1$.

Our first main theorem is the following one.

Theorem 1.1. If the differential system (1.1) with $n>2$ has a Weierstrass inverse integrating factor of the form (1.4) with $s=n$, then

$$
\begin{gathered}
V_{i}(x)=\frac{a_{i}(x)}{a_{n}(x)} \text { for } i=2, \ldots, n-1, \\
V_{1}(x)=\frac{a_{1}(x)}{a_{n}(x)}+\frac{1}{(n-1) a_{n}(x)} \frac{d}{d x} \frac{a_{n-1}(x)}{a_{n}(x)}+\frac{(n-2) a_{1}(x) a_{n-1}(x)}{(n-1) a_{n}^{2}(x)}
\end{gathered}
$$

and

$$
V_{0}(x)=\frac{a_{0}(x)}{a_{n}(x)}+\frac{1}{n a_{n}(x)} \frac{d}{d x} \frac{a_{n-2}(x)}{a_{n}(x)}-\frac{(n-2) a_{n-1}(x)}{n(n-1) a_{n}^{2}(x)} \frac{d}{d x} \frac{a_{n-1}(x)}{a_{n}(x)}-\frac{(n-2)^{2} a_{1}(x) a_{n-1}^{2}(x)}{n(n-1) a_{n}^{3}(x)} .
$$

The proof of Theorem 1.1 is given in section 2 .

The following result characterizes the existence of Weierstrass inverse integrating factors of the differential systems (1.1) when $n=2$.

Theorem 1.2. System (1.1) with $n=2$ has a Weierstrass inverse integrating factor of the form (1.4) with $s=2$, if and only if either $a_{0}(x)=a_{1}(x)=0$, and then $V=y^{2}$; 
or $a_{0}(x)=0$ and $a_{1}(x) \neq 0$, and then $V_{0}=0$ and

$$
V_{1}(x)=-e^{\int a_{2}(x) d x} \int a_{1}(x) e^{-\int a_{2}(s) d s} d x ;
$$

or $a_{0}(x) \neq 0$ and $a_{1}(x)=0$, and then $V_{1}=0$, and

$$
V_{0}(x)=-2 e^{2 \int a_{2}(x) d x} \int a_{0}(x) e^{-2 \int a_{2}(s) d s} d x ;
$$

or $a_{0}(x) a_{1}(x) \neq 0$ with $a_{0}(x) a_{1}(x) a_{2}(x)+a_{0}(x) a_{1}^{\prime}(x)-a_{0}^{\prime}(x) \neq 0$ and

$$
\begin{array}{r}
a_{0}^{\prime}(x) a_{1}(x)^{2} a_{2}(x)+a_{0}(x) a_{1}(x)^{2} a_{2}^{\prime}(x)-a_{0}^{\prime \prime}(x) a_{1}(x)^{2}-3 a_{0}(x) a_{1}^{\prime}(x)^{2} \\
+3 a_{0}^{\prime}(x) a_{1}(x) a_{1}^{\prime}(x)+a_{0}(x) a_{1}(x) a_{1}^{\prime \prime}(x)-2 a_{0}(x) a_{1}(x) a_{1}^{\prime}(x) a_{2}(x)=0,
\end{array}
$$

then

$$
V_{0}(x)=\frac{a_{1}(x) a_{0}^{2}(x)}{a_{0}(x) a_{1}(x) a_{2}(x)+a_{0}(x) a_{1}^{\prime}(x)-a_{0}^{\prime}(x) a_{1}(x)}
$$

and

$$
V_{1}(x)=\frac{a_{1}^{2}(x) a_{0}(x)}{a_{0}(x) a_{1}(x) a_{2}(x)+a_{0}(x) a_{1}^{\prime}(x)-a_{0}^{\prime}(x) a_{1}(x)} .
$$

The proof of Theorem 1.2 is given in section 3. After the proof of this theorem we apply it to two examples. One satisfying the assumptions of the theorem, and consequently having an inverse integrating factor of the form (1.4) with $s=2$, and another which does not satisfy the hypotheses of the theorem and consequently it has not an inverse integrating factor of the form (1.4) with $s=2$.

The second main result of the paper is the following.

Theorem 1.3. System (1.1) with $n \geq 3$ has a Weierstrass inverse integrating factor of the form in (1.4) if and only if the following conditions hold:

$$
\begin{aligned}
a_{k}(0)=0 \text { for } k=2, \ldots & , n-1, \quad a_{1}(0)=-\frac{a_{n-1}^{\prime}(0)}{(n-1) a_{n}(0)}, \quad a_{0}(0)=-\frac{a_{n-2}^{\prime}(0)}{n a_{n}(0)}, \\
\frac{d}{d x} \frac{a_{n-k-1}(x)}{a_{n}(x)}= & \frac{n-k+1}{n a_{n}(x)} a_{n-k+1}(x) \frac{d}{d x} \frac{a_{n-2}(x)}{a_{n}(x)} \\
& -\frac{(n-k+1)(n-2) a_{n-1}(x)}{n(n-1) a_{n}(x)^{2}} a_{n-k+1}(x) \frac{d}{d x} \frac{a_{n-1}(x)}{a_{n}(x)} \\
& -\frac{(n-k+1)(n-2)^{2} a_{1}(x) a_{n-1}^{2}(x)}{n(n-1) a_{n}^{3}(x)} a_{n-k+1}(x) .
\end{aligned}
$$

for $k=0, \ldots, n-3$,

$$
\begin{aligned}
& \frac{d}{d x}\left(\frac{a_{1}(x)}{a_{n}(x)}+\frac{1}{(n-1) a_{n}(x)} \frac{d}{d x} \frac{a_{n-1}(x)}{a_{n}(x)}+\frac{(n-2) a_{1}(x) a_{n-1}(x)}{(n-1) a_{n}^{2}(x)}\right) \\
& =3 a_{3}(x)\left(\frac{1}{n a_{n}(x)} \frac{d}{d x} \frac{a_{n-2}(x)}{a_{n}(x)}-\frac{(n-2)}{n(n-1)} \frac{a_{n-1}(x)}{a_{n}^{2}(x)} \frac{d}{d x} \frac{a_{n-1}(x)}{a_{n}(x)}-\frac{(n-2)^{2} a_{1}(x) a_{n-1}^{2}(x)}{n(n-1) a_{n}^{3}(x)}\right) \\
& \quad+\frac{a_{2}(x)}{(n-1) a_{n}(x)} \frac{d}{d x} \frac{a_{n-1}(x)}{a_{n}(x)}+\frac{(n-2) a_{1}(x) a_{2}(x) a_{n-1}(x)}{(n-1) a_{n}^{2}(x)}
\end{aligned}
$$




$$
\begin{aligned}
& \frac{d}{d x}\left(\frac{a_{0}(x)}{a_{n}(x)}+\frac{1}{n a_{n}(x)} \frac{d}{d x} \frac{a_{n-2}(x)}{a_{n}(x)}-\frac{(n-2) a_{n-1}(x)}{n(n-1) a_{n}^{2}(x)} \frac{d}{d x} \frac{a_{n-1}(x)}{a_{n}(x)}-\frac{(n-2)^{2} a_{1}(x) a_{n-1}^{2}(x)}{n(n-1) a_{n}^{3}(x)}\right) \\
& =2 a_{2}(x)\left(\frac{1}{n a_{n}(x)} \frac{d}{d x} \frac{a_{n-2}(x)}{a_{n}(x)}-\frac{(n-2)}{n(n-1)} \frac{a_{n-1}(x)}{a_{n}(x)^{2}} \frac{d}{d x} \frac{a_{n-1}(x)}{a_{n}(x)}-\frac{(n-2)^{2} a_{1}(x) a_{n-1}^{2}(x)}{n(n-1) a_{n}^{3}(x)}\right)
\end{aligned}
$$

and

$$
\begin{aligned}
& \frac{a_{0}(x)}{(n-1)} \frac{d}{d x} \frac{a_{n-1}(x)}{a_{n}(x)}+\frac{(n-2) a_{0}(x) a_{1}(x) a_{n-1}(x)}{(n-1) a_{n}(x)} \\
& =\frac{a_{1}(x)}{n} \frac{d}{d x} \frac{a_{n-2}(x)}{a_{n}(x)}-\frac{(n-2)}{n(n-1)} \frac{a_{1}(x) a_{n-1}(x)}{a_{n}(x)} \frac{d}{d x} \frac{a_{n-1}(x)}{a_{n}(x)}-\frac{(n-2)^{2} a_{1}^{2}(x) a_{n-1}^{2}(x)}{n(n-1) a_{n}^{2}(x)} .
\end{aligned}
$$

The proof of Theorem 1.3 is given in section 4 .

In [9] and [10] the Weierstrass integrability has been characterized for the particular differential systems (1.1) with $n=3,4$.

\section{Proof of Proposition 1.1 and Theorem 1.1}

Proof of Proposition 1.1. If the differential system (1.1) has a first integral of the form (1.3) we get

$$
\sum_{i=0}^{s-1} H_{i}^{\prime}(x) y^{i+1}+\sum_{i=0}^{s} i H_{i}(x) y^{i-1}\left(a_{n}(x) y^{n}+a_{n-1}(x) y^{n-1}+\cdots+a_{1}(x) y+a_{0}(x)\right)=0 .
$$

The highest power is $y^{s+n-1}$ (since $n \geq 2$ ) and its coefficient is $s a_{n}(x)=0$. Since we are assuming that $a_{n}(x) \neq 0$ we get $s=0$. So $H=H_{0}(x)$. Then, from (2.1), it follows that $H_{0}^{\prime}(x)=0$, that is $H=H_{0}$, a constant in contradiction with the fact that $H$ is a first integral.

Imposing that system (1.1) with $n>2$ has an inverse integrating factor of the form (1.4) we obtain a polynomial in the variable $y$ whose coefficients must be zero. Hence we get that

$$
\begin{aligned}
& \sum_{i=0}^{s-1} V_{i}^{\prime}(x) y^{i+1}+\sum_{i=0}^{s} i V_{i}(x) y^{i-1}\left(a_{n}(x) y^{n}+a_{n-1}(x) y^{n-1}+\cdots+a_{1}(x) y+a_{0}(x)\right) \\
& =\left(n a_{n}(x) y^{n-1}+(n-1) a_{n-1}(x) y^{n-2}+\cdots+2 a_{2}(x) y+a_{1}(x)\right)\left(\sum_{i=0}^{s} V_{i}(x) y^{i}\right) .
\end{aligned}
$$

Now computing the terms in (2.2) with $y^{s+n-1}$ with $n \geq 2$ we get

$$
s V_{s}(x) a_{n}(x)=n V_{s}(x) a_{n}(x) .
$$

In short $s=n \geq 2$ because $V_{s}(0) a_{n}(0)=a_{n}(0) \neq 0$. Now we state and prove some auxiliary results.

Lemma 2.1. Equation (2.2) can be written as

$$
\sum_{i=0}^{n-1} V_{i}^{\prime}(x) y^{i+1}+a_{0}(x) \sum_{i=0}^{n-1}(i+1) V_{i+1}(x) y^{i}+\sum_{l=0}^{2 n-2} y^{l} \sum_{i=\max \{0, l+1-n\}}^{\min \{l, n\}}(2 i-1-l) V_{i}(x) a_{l-i+1}(x) .
$$


Proof. Since $V_{n}(x)=1$ equation (2.2) can be written as

$$
\begin{aligned}
0= & \sum_{i=0}^{n-1} V_{i}^{\prime}(x) y^{i+1}+a_{0}(x) \sum_{i=1}^{n} i V_{i}(x) y^{i-1} \\
& +\sum_{i=0}^{n} i V_{i}(x) y^{i-1}\left(a_{n}(x) y^{n}+a_{n-1}(x) y^{n-1}+\cdots+a_{1}(x) y\right) \\
& \quad-\left(n a_{n}(x) y^{n-1}+(n-1) a_{n-1}(x) y^{n-2}+\cdots+2 a_{2}(x) y+a_{1}(x)\right)\left(\sum_{i=0}^{n} V_{i}(x) y^{i}\right) \\
= & \sum_{i=0}^{n-1} V_{i}^{\prime}(x) y^{i+1}+a_{0}(x) \sum_{i=0}^{n-1}(i+1) V_{i+1}(x) y^{i} \\
& +\sum_{i=0}^{n} i V_{i}(x) y^{i-1}\left(a_{n}(x) y^{n}+a_{n-1}(x) y^{n-1}+\cdots+a_{1}(x) y\right) \\
& -\sum_{i=0}^{n} V_{i}(x) y^{i-1}\left(n a_{n}(x) y^{n}+(n-1) a_{n-1}(x) y^{n-1}+\cdots+a_{1}(x) y\right) \\
= & \sum_{i=0}^{n-1} V_{i}^{\prime}(x) y^{i+1}+a_{0}(x) \sum_{i=0}^{n-1}(i+1) V_{i+1}(x) y^{i} \\
& +\sum_{i=0}^{n} V_{i}(x) y^{i-1}\left((i-n) a_{n}(x) y^{n}+(i-n+1) a_{n-1}(x) y^{n-1}+\cdots+(i-1) a_{1}(x) y\right) .
\end{aligned}
$$

We can write the last sum in (2) as

$$
\begin{aligned}
& \sum_{i=0}^{n} V_{i}(x) y^{i-1}\left((i-n) a_{n}(x) y^{n}+(i-n+1) a_{n-1}(x) y^{n-1}+\cdots+(i-1) a_{1}(x) y\right) \\
& =\sum_{j=0}^{n-1} \sum_{i=0}^{n}(i-n+j) V_{i}(x) a_{n-j}(x) y^{n+i-1-j} \\
& =\sum_{l=0}^{2 n-2} y^{l} \sum_{i=\max \{0, l+1-n\}}^{\min \{l, n\}}(2 i-1-l) V_{i}(x) a_{l-i+1}(x) .
\end{aligned}
$$

Now the proof follows immediately from (2) and (2.5).

Let

$$
S_{n, l}(x)=\sum_{j=1}^{l-1}(l-2 j) a_{n-j}(x) a_{n-l+j}(x) .
$$

Note that $l \leq n+1$. The following two lemmas are the Lemmas 4 and 5 of [9], respectively.

Lemma 2.2. We have that $S_{n, l}(x)=0$. 
Lemma 2.3. For $k=2, \ldots, n-1$ we have that

$$
V_{k}(x)=\frac{a_{k}(x)}{a_{n}(x)}
$$

Lemma 2.4. We have that

$$
V_{1}(x)=\frac{a_{1}(x)}{a_{n}(x)}+\frac{1}{(n-1) a_{n}(x)} \frac{d}{d x} \frac{a_{n-1}(x)}{a_{n}(x)}+\frac{(n-2) a_{1}(x) a_{n-1}(x)}{(n-1) a_{n}^{2}(x)} .
$$

Proof. We compute in (2.2) with $s=n$ the coefficient of $y^{n}$. By Lemma 2.1 with $l=n$ we get

$$
V_{n-1}^{\prime}(x)+\sum_{i=1}^{n}(2 i-1-n) V_{i}(x) a_{n-i+1}(x)=0 .
$$

Now, using Lemma 2.3 we get

$$
\begin{aligned}
0= & V_{n-1}^{\prime}(x)+(1-n) V_{1}(x) a_{n}(x)+(n-1) V_{n}(x) a_{1}(x)+\sum_{i=2}^{n-1}(2 i-1-n) V_{i}(x) a_{n-i}(x) \\
= & V_{n-1}^{\prime}(x)+(1-n) V_{1}(x) a_{n}(x)+(n-1) a_{1}(x)+\frac{1}{a_{n}(x)} \sum_{i=2}^{n-1}(2 i-n) a_{i}(x) a_{n-i}(x) \\
& -\frac{(2-n) a_{1}(x) a_{n-1}(x)}{a_{n}(x)} \\
= & V_{n-1}^{\prime}(x)+(1-n) V_{1}(x) a_{n}(x)+(n-1) a_{1}(x)-\frac{S_{n, n}(x)}{a_{n}(x)}-\frac{(2-n) a_{1}(x) a_{n-1}(x)}{a_{n}(x)} \\
= & V_{n-1}^{\prime}(x)+(1-n) V_{1}(x) a_{n}(x)+(n-1) a_{1}(x)-\frac{(2-n) a_{1}(x) a_{n-1}(x)}{a_{n}(x)}
\end{aligned}
$$

where in the last equality we have used that $S_{n, n}(x)=0$, see Lemma 2.2. Therefore,

$$
\begin{aligned}
V_{1}(x) & =\frac{1}{(n-1) a_{n}(x)}\left((n-1) a_{1}(x)+V_{n-1}^{\prime}(x)+\frac{(n-2) a_{1}(x) a_{n-1}(x)}{a_{n}(x)}\right) \\
& =\frac{a_{1}(x)}{a_{n}(x)}+\frac{1}{(n-1) a_{n}(x)} \frac{d}{d x} \frac{a_{n-1}(x)}{a_{n}(x)}+\frac{(n-2) a_{1}(x) a_{n-1}(x)}{a_{n}(x)} .
\end{aligned}
$$

This concludes the proof of the lemma.

Lemma 2.5. We have that

$$
V_{0}(x)=\frac{a_{0}(x)}{a_{n}(x)}+\frac{1}{n a_{n}(x)} \frac{d}{d x} \frac{a_{n-2}(x)}{a_{n}(x)}-\frac{(n-2) a_{n-1}(x)}{n(n-1) a_{n}^{2}(x)} \frac{d}{d x} \frac{a_{n-1}(x)}{a_{n}(x)}-\frac{(n-2)^{2} a_{1}(x) a_{n-1}^{2}(x)}{n(n-1) a_{n}^{3}(x)} .
$$

Proof. We compute in (2.2) with $s=n$ the coefficient of $y^{n-1}$. By Lemma 2.1 with $l=n-1$ we get

$$
V_{n-2}^{\prime}(x)+n a_{0}(x) V_{n}(x)+\sum_{i=0}^{n-1}(2 i-n) V_{i}(x) a_{n-i}(x)=0
$$


Now using Lemma 2.3 we get

$$
\begin{aligned}
& V_{n-2}^{\prime}(x)+n a_{0}(x) V_{n}(x)-n V_{0}(x) a_{n}(x)+(2-n) V_{1}(x) a_{n-1}(x)+\sum_{i=2}^{n-1}(2 i-n) V_{i}(x) a_{n-i}(x) \\
& =V_{n-2}^{\prime}(x)+n a_{0}(x)-n V_{0}(x) a_{n}(x)+(2-n) V_{1}(x) a_{n-1}(x)+\frac{1}{a_{n}(x)} \sum_{i=2}^{n-1}(2 i-n) a_{i}(x) a_{n-i}(x) \\
& =V_{n-2}^{\prime}(x)+n a_{0}(x)-n V_{0}(x) a_{n}(x)+(2-n) V_{1}(x) a_{n-1}(x)-\frac{S_{n, n}(x)}{a_{n}(x)}+\frac{(n-2) a_{1}(x) a_{n-1}(x)}{a_{n}(x)} \\
& =V_{n-2}^{\prime}(x)+n a_{0}(x)-n V_{0}(x) a_{n}(x)+(2-n) V_{1}(x) a_{n-1}(x)+\frac{(n-2) a_{1}(x) a_{n-1}(x)}{a_{n}(x)}=0 .
\end{aligned}
$$

Therefore

$$
\begin{aligned}
V_{0}(x)= & \frac{1}{n a_{n}(x)}\left(n a_{0}(x)+V_{n-2}^{\prime}(x)-(n-2)\left(V_{1}(x) a_{n-1}(x)-\frac{a_{1}(x) a_{n-1}(x)}{a_{n}(x)}\right)\right. \\
= & \frac{a_{0}(x)}{a_{n}(x)}+\frac{1}{n a_{n}(x)} \frac{d}{d x} \frac{a_{n-2}(x)}{a_{n}(x)}-\frac{n-2}{n a_{n}(x)}\left(\frac{a_{1}(x) a_{n-1}(x)}{a_{n}(x)}\right. \\
& \left.+\frac{a_{n-1}(x)}{(n-1) a_{n}(x)} \frac{d}{d x} \frac{a_{n-1}(x)}{a_{n}(x)}+\frac{(n-2) a_{1}(x) a_{n-1}^{2}(x)}{(n-1) a_{n}^{2}(x)}-\frac{a_{1}(x) a_{n-1}(x)}{a_{n}(x)}\right) \\
= & \frac{a_{0}(x)}{a_{n}(x)}+\frac{1}{n a_{n}(x)} \frac{d}{d x} \frac{a_{n-2}(x)}{a_{n}(x)}-\frac{(n-2) a_{n-1}(x)}{n(n-1) a_{n}^{2}(x)} \frac{d}{d x} \frac{a_{n-1}(x)}{a_{n}(x)}-\frac{(n-2)^{2} a_{1}(x) a_{n-1}^{2}(x)}{n(n-1) a_{n}^{3}(x)} .
\end{aligned}
$$

This concludes the proof of the lemma.

Proof of Theorem 1.1. The proof of Theorem 1.1 is an immediate consequence of Lemmas 2.3, 2.4 and 2.5 .

\section{Proof of Theorem 1.2}

In this section we consider system (1.1) with $n=2$. In this case (2.2) with $s=2$ and $V=V_{0}(x)+$ $V_{1}(x) y+y^{2}$ becomes

$$
\begin{aligned}
& V_{0}^{\prime}(x) y+V_{1}^{\prime}(x) y^{2}+\left(V_{1}(x)+2 y\right)\left(a_{2}(x) y^{2}+a_{1}(x) y+a_{0}(x)\right) \\
& =\left(2 a_{2}(x) y+a_{1}(x)\right)\left(V_{0}(x)+V_{1}(x) y+y^{2}\right),
\end{aligned}
$$

that is,

$$
\begin{aligned}
a_{0}(x) V_{1}(x)-a_{1}(x) V_{0}(x) & =0, \\
V_{0}^{\prime}(x)-2 a_{2}(x) V_{0}(x)+2 a_{0}(x) & =0, \\
V_{1}^{\prime}(x)-a_{2}(x) V_{1}(x)+a_{1}(x) & =0 .
\end{aligned}
$$

Solving the third equation in system (3.2) we get

$$
V_{1}(x)=e^{\int a_{2}(x) d x} c_{1}-e^{\int a_{2}(x) d x} \int a_{1}(x) e^{-\int a_{2}(s) d s} d x, \quad c_{1} \in \mathbb{C} .
$$

Since we are interesting in finding one Weierstrass inverse integrating and not all, we choose $c_{1}=0$. 
Moreover, solving the second equation in system (3.2) we get

$$
V_{0}(x)=e^{2 \int a_{2}(x) d x} c_{0}-2 e^{2 \int a_{2}(x) d x} \int a_{0}(x) e^{-2 \int a_{2}(s) d s} d x .
$$

Again since we are interesting in finding one Weierstrass inverse integrating and not all, we choose $c_{0}=0$.

Imposing the values of $V_{1}(x)$ and $V_{0}(x)$ in the first equation in (3.2) we obtain

$$
a_{0}(x) \int a_{1}(x) e^{-\int a_{2}(s) d s} d x=2 a_{1}(x) e^{\int a_{2}(x) d x} \int a_{0}(x) e^{-2 \int a_{2}(s) d s} d x .
$$

It is clear that if $a_{0}(x)=0$ (respectively $a_{1}(x)=0$ ), then $V_{0}(x)=0$ (respectively $V_{1}(x)=0$ ). Therefore the statement in the theorem for the cases $a_{0}(x)=a_{1}(x)=0, a_{0}(x)=0$ and $a_{1}(x) \neq 0$, and $a_{0}(x) \neq 0$ and $a_{1}(x)=0$ follow.

Now assume that $a_{0}(x) a_{1}(x) \neq 0$. In this case we will rewrite (3.3), (3.4) and (3.5) in a more compact form, without quadratures. It follows from the first relation in (3.2) that

$$
V_{1}(x)=\frac{a_{1}(x)}{a_{0}(x)} V_{0}(x)
$$

and

$$
a_{0}^{\prime}(x) V_{1}(x)+a_{0}(x) V_{1}^{\prime}(x)-a_{1}^{\prime}(x) V_{0}(x)-a_{1}(x) V_{0}^{\prime}(x)=0 .
$$

Now using (3.6) and the second and third relations in (3.2) we get

$$
\begin{aligned}
& a_{0}^{\prime}(x) \frac{a_{1}(x)}{a_{0}(x)} V_{0}(x)+a_{2}(x) a_{1}(x) V_{0}(x)-a_{0}(x) a_{1}(x)-a_{1}^{\prime}(x) V_{0}(x)-2 a_{1}(x) a_{2}(x) V_{0}(x)+ \\
& 2 a_{1}(x) a_{0}(x)=V_{0}(x)\left(a_{0}^{\prime}(x) \frac{a_{1}(x)}{a_{0}(x)}-a_{2}(x) a_{1}(x)-a_{1}^{\prime}(x)\right)+a_{1}(x) a_{0}(x)=0,
\end{aligned}
$$

and thus

$$
V_{0}(x)=\frac{a_{1}(x) a_{0}^{2}(x)}{a_{0}(x) a_{1}(x) a_{2}(x)+a_{0}(x) a_{1}^{\prime}(x)-a_{0}^{\prime}(x) a_{1}(x)},
$$

with $a_{0}(x) a_{1}(x) a_{2}(x)+a_{0}(x) a_{1}^{\prime}(x)-a_{0}^{\prime}(x) \neq 0$, otherwise there is no solution. Again from (3.6) we get

$$
V_{1}(x)=\frac{a_{1}^{2}(x) a_{0}(x)}{a_{0}(x) a_{1}(x) a_{2}(x)+a_{0}(x) a_{1}^{\prime}(x)-a_{0}^{\prime}(x) a_{1}(x)}
$$

Now we write (3.5) as

$$
\int a_{1}(x) e^{-\int a_{2}(s) d s} d x=2 \frac{a_{1}(x)}{a_{0}(x)} e^{\int a_{2}(x) d x} \int a_{0}(x) e^{-2 \int a_{2}(s) d s} d x .
$$

Taking derivatives in $x$ we get

$$
\begin{aligned}
a_{1}(x) e^{-\int a_{2}(x) d x}= & 2 \frac{a_{1}^{\prime}(x) a_{0}(x)-a_{1}(x) a_{0}^{\prime}(x)}{a_{0}^{2}(x)} e^{\int a_{2}(x) d x} \int a_{0}(x) e^{-2 \int a_{2}(s) d s} d x \\
& +2 \frac{a_{1}(x) a_{2}(x)}{a_{0}(x)} e^{\int a_{2}(x) d x} \int a_{0}(x) e^{-2 \int a_{2}(s) d s} d x+2 a_{1}(x) e^{-\int a_{2}(x) d x} .
\end{aligned}
$$


Hence

$$
\begin{aligned}
-a_{1}(x) e^{-\int a_{2}(x) d x}= & 2 \frac{a_{1}^{\prime}(x) a_{0}(x)-a_{1}(x) a_{0}^{\prime}(x)+a_{0}(x) a_{1}(x) a_{2}(x)}{a_{0}^{2}(x)} \\
& e^{\int a_{2}(x) d x} \int a_{0}(x) e^{-2 \int a_{2}(s) d s} d x,
\end{aligned}
$$

or equivalently

$$
\frac{a_{1}(x) a_{0}^{2}(x) e^{-2 \int a_{2}(x) d x}}{a_{1}^{\prime}(x) a_{0}(x)-a_{1}(x) a_{0}^{\prime}(x)+a_{0}(x) a_{1}(x) a_{2}(x)}=-2 \int a_{0}(x) e^{-2 \int a_{2}(s) d s} d x .
$$

Taking again derivatives in $x$ we get

$$
\begin{gathered}
a_{0}(x)\left(a_{2}(x) a_{0}^{\prime}(x) a_{1}(x)^{2}+a_{0}(x) a_{2}^{\prime}(x) a_{1}(x)^{2}-a_{0}^{\prime \prime}(x) a_{1}(x)^{2}-2 a_{0}(x) a_{2}(x) a_{1}^{\prime}(x) a_{1}(x)+\right. \\
\left.3 a_{0}^{\prime}(x) a_{1}^{\prime}(x) a_{1}(x)+a_{0}(x) a_{1}^{\prime \prime}(x) a_{1}(x)-3 a_{0}(x) a_{1}^{\prime}(x)^{2}\right)=0 .
\end{gathered}
$$

This concludes the proof of Theorem 1.2.

Example 1: We apply Theorem 1.2 to the differential system

$$
\dot{x}=y, \quad \dot{y}=y^{2}+(b+a x) y-(b+a x)(a+b+a x),
$$

with $a b \neq 0$. Then

$$
a_{0}(x)=(b+a x)(a+b+a x) \neq 0, \quad a_{1}(x)=b+a x \neq 0, \quad a_{2}(x)=1,
$$

satisfy the assumptions of Theorem 1.2 corresponding to the case $a_{0}(x) a_{1}(x) \neq 0$, and from this theorem this differential system has the inverse integrating factor

$$
V(x, y)=y^{2}+(a+b+a x) y-(a+b+a x)^{2},
$$

as it is easy to check directly from the definition of inverse integrating factor. Once we know an inverse integrating factor we can compute a first integral, and we obtain

$$
\begin{gathered}
H=-10 x-(-5+\sqrt{5}) \log ((-1+\sqrt{5}) b+(-1+\sqrt{5}) a(x+1)-2 y)+ \\
(5+\sqrt{5}) \log (\sqrt{5} b+b+(1+\sqrt{5}) a(x+1)+2 y) .
\end{gathered}
$$

Example 2: We consider the differential system

$$
\dot{x}=y, \quad \dot{y}=y^{2}+2 y+x,
$$

then

$$
a_{0}(x)=1, \quad a_{1}(x)=2, \quad a_{2}(x)=x,
$$

and this system does not satisfy the assumptions of Theorem 1.2 corresponding to the case $a_{0}(x) a_{1}(x) \neq 0$. Now we shall prove that this differential system has not an inverse integrating factor 
of the form (1.4) with $s=2$. Indeed, if it has an inverse integrating factor $V=V_{0}(x)+V_{1}(x) y+y^{2}$, then from the second and third equations of (3.2) we obtain

$$
\begin{aligned}
& V_{0}(x)=-2\left(-\frac{x}{2}-\frac{1}{4}\right)+c_{0} e^{2 x}, \\
& V_{1}(x)=2+c_{1} e^{x},
\end{aligned}
$$

respectively. Finally from the first equation of (3.2) we get the contradiction

$$
-1-2 c_{0} e^{2 x}+c_{1} x e^{x}=0 .
$$

\section{Proof of Theorem 1.3}

Lemma 4.1. For $k=2, \ldots, n-1$ we have that $a_{k}(0)=0$ and

$$
a_{1}(0)=-\frac{a_{n-1}^{\prime}(0)}{(n-1) a_{n}(0)}, \quad a_{0}(0)=-\frac{a_{n-2}^{\prime}(0)}{n a_{n}(0)} .
$$

Proof. Since $V_{k}(0)=0$ for $k=0, \ldots, n-1$ and $a_{n}(0) \neq 0$, it follows from Lemma 2.3 that $a_{k}(0)=0$ for $k=2, \ldots, n-1$.

Now using that $V_{1}(0)=0$ it follows from Lemma 2.4 and the explanation above (note that $\left.a_{n-1}(0)=0\right)$ that

$$
0=V_{1}(0)=\frac{a_{1}(0)}{a_{n}(0)}+\frac{a_{n-1}^{\prime}(0)}{(n-1) a_{n}^{2}(0)} .
$$

¿From here the expression of $a_{1}(0)$ follows. Now using that $V_{0}(0)=0$ and $a_{n-1}(0)=a_{n-2}(0)=0$, it follows from Lemma 2.5 that

$$
0=V_{0}(0)=\frac{a_{0}(0)}{a_{n}(0)}+\frac{a_{n-2}^{\prime}(0)}{n a_{n}(0)^{2}}
$$

which completes the proof of the lemma.

Lemma 4.2. For $k=0, \ldots, n-3$, we have

$$
\begin{aligned}
\frac{d}{d x} \frac{a_{n-k-1}(x)}{a_{n}(x)}= & \frac{n-k+1}{n a_{n}(x)} a_{n-k+1}(x) \frac{d}{d x} \frac{a_{n-2}(x)}{a_{n}(x)} \\
& -\frac{(n-k+1)(n-2) a_{n-1}(x)}{n(n-1) a_{n}(x)^{2}} a_{n-k+1}(x) \frac{d}{d x} \frac{a_{n-1}(x)}{a_{n}(x)} \\
& -\frac{(n-k+1)(n-2)^{2} a_{1}(x) a_{n-1}^{2}(x)}{n(n-1) a_{n}^{3}(x)} a_{n-k+1}(x) .
\end{aligned}
$$


Proof. We compute in (2.2) with $s=n$ the coefficients of $y^{n-k}$ with $k=0, \ldots, n-3$. By Lemma 2.1 with $l=n-k$ we get

$$
V_{n-k-1}^{\prime}(x)+(n-k+1) a_{0}(x) V_{n-k+1}(x)+\sum_{i=0}^{n-k}(2 i-1-n+k) V_{i}(x) a_{n-k-i+1}(x)=0 .
$$

Using Lemma 2.5 we get that (4.1) becomes

$$
\begin{aligned}
0= & \frac{d}{d x} \frac{a_{n-k-1}(x)}{a_{n}(x)}+(n-k+1) a_{0}(x) \frac{a_{n-k+1}(x)}{a_{n}(x)}+\sum_{i=0}^{n-k}(2 i-1-n+k) V_{i}(x) a_{n-k-i+1}(x) \\
= & \frac{d}{d x} \frac{a_{n-k-1}(x)}{a_{n}(x)}+(n-k+1) a_{0}(x) \frac{a_{n-k+1}(x)}{a_{n}(x)}-(n-k+1) V_{0}(x) a_{n-k+1}(x) \\
& +\frac{1}{a_{n}(x)} \sum_{i=1}^{n-k}(2 i-1-n+k) a_{i}(x) a_{n-k-i+1}(x) \\
= & \frac{d}{d x} \frac{a_{n-k-1}(x)}{a_{n}(x)}+(n-k+1) a_{0}(x) \frac{a_{n-k+1}(x)}{a_{n}(x)}-(n-k+1) V_{0}(x) a_{n-k+1}(x) \\
& -\frac{S_{n-k+1, n-k+1}(x)}{a_{n}(x)} .
\end{aligned}
$$

Now using Lemma 2.2 we get

$$
\frac{d}{d x} \frac{a_{n-k-1}(x)}{a_{n}(x)}+(n-k+1) a_{0}(x) \frac{a_{n-k+1}(x)}{a_{n}(x)}-(n-k+1) V_{0}(x) a_{n-k+1}(x)=0,
$$

and by Lemma 2.5 , we deduce that

$$
\begin{aligned}
0= & \frac{d}{d x} \frac{a_{n-k-1}(x)}{a_{n}(x)}+(n-k+1) a_{0}(x) \frac{a_{n-k+1}(x)}{a_{n}(x)}-(n-k+1) \frac{a_{0}(x)}{a_{n}(x)} a_{n-k+1}(x) \\
& -\frac{n-k+1}{n a_{n}(x)} a_{n-k+1}(x) \frac{d}{d x} \frac{a_{n-2}(x)}{a_{n}(x)}+\frac{(n-k+1)(n-2) a_{n-1}(x)}{n(n-1) a_{n}(x)^{2}} a_{n-k+1}(x) \frac{d}{d x} \frac{a_{n-1}(x)}{a_{n}(x)} \\
& +\frac{(n-k+1)(n-2)^{2} a_{1}(x) a_{n-1}^{2}(x)}{n(n-1) a_{n}^{3}(x)} a_{n-k+1}(x) .
\end{aligned}
$$

This concludes the proof of the lemma.

Lemma 4.3. We have

$$
\begin{aligned}
\frac{d V_{1}(x)}{d x}= & 3 a_{3}(x)\left(\frac{1}{n a_{n}(x)} \frac{d}{d x} \frac{a_{n-2}(x)}{a_{n}(x)}-\frac{(n-2)}{n(n-1)} \frac{a_{n-1}(x)}{a_{n}^{2}(x)} \frac{d}{d x} \frac{a_{n-1}(x)}{a_{n}(x)}-\frac{(n-2)^{2} a_{1}(x) a_{n-1}^{2}(x)}{n(n-1) a_{n}^{3}(x)}\right) \\
& +\frac{a_{2}(x)}{(n-1) a_{n}(x)} \frac{d}{d x} \frac{a_{n-1}(x)}{a_{n}(x)}+\frac{(n-2) a_{1}(x) a_{2}(x) a_{n-1}(x)}{(n-1) a_{n}^{2}(x)} .
\end{aligned}
$$

where $V_{1}(x)$ is given in Lemma 2.4. 
Proof. We compute in (2.2) with $s=n$ the coefficients of $y^{2}$ and we have

$$
V_{1}^{\prime}(x)+3 V_{3}(x) a_{0}(x)-3 V_{0}(x) a_{3}(x)-V_{1}(x) a_{2}(x)+V_{2}(x) a_{1}(x)=0 .
$$

Using Lemmas 2.3, 2.4 and 2.5 we get

$$
\begin{aligned}
0= & \frac{d V_{1}(x)}{d x}+\frac{3 a_{3}(x)}{a_{n}(x)} a_{0}(x)-3 a_{3}(x)\left(\frac{a_{0}(x)}{a_{n}(x)}+\frac{1}{n a_{n}(x)} \frac{d}{d x} \frac{a_{n-2}(x)}{a_{n}(x)}\right. \\
& \left.-\frac{(n-2) a_{n-1}(x)}{n(n-1) a_{n}^{2}(x)} \frac{d}{d x} \frac{a_{n-1}(x)}{a_{n}(x)}-\frac{(n-2)^{2} a_{1}(x) a_{n-1}^{2}(x)}{n(n-1) a_{n}^{3}(x)}\right) \\
& -a_{2}(x)\left(\frac{a_{1}(x)}{a_{n}(x)}+\frac{1}{(n-1) a_{n}(x)} \frac{d}{d x} \frac{a_{n-1}(x)}{a_{n}(x)}+\frac{(n-2) a_{1}(x) a_{n-1}(x)}{(n-1) a_{n}^{2}(x)}\right)+\frac{a_{2}(x)}{a_{n}(x)} a_{1}(x) \\
= & \frac{d V_{1}(x)}{d x}-3 a_{3}(x)\left(\frac{1}{n a_{n}(x)} \frac{d}{d x} \frac{a_{n-2}(x)}{a_{n}(x)}-\frac{(n-2)}{n(n-1)} \frac{a_{n-1}(x)}{a_{n}^{2}(x)} \frac{d}{d x} \frac{a_{n-1}(x)}{a_{n}(x)}\right. \\
& \left.-\frac{(n-2)^{2} a_{1}(x) a_{n-1}^{2}(x)}{n(n-1) a_{n}^{3}(x)}\right)-\frac{a_{2}(x)}{(n-1) a_{n}(x)} \frac{d}{d x} \frac{a_{n-1}(x)}{a_{n}(x)}-\frac{(n-2) a_{1}(x) a_{2}(x) a_{n-1}(x)}{(n-1) a_{n}^{2}(x)} .
\end{aligned}
$$

This concludes the proof of the lemma.

Lemma 4.4. We have that

$$
\begin{aligned}
\frac{d V_{0}(x)}{d x}= & 2 a_{2}(x)\left(\frac{1}{n a_{n}(x)} \frac{d}{d x} \frac{a_{n-2}(x)}{a_{n}(x)}-\frac{(n-2)}{n(n-1)} \frac{a_{n-1}(x)}{a_{n}(x)^{2}} \frac{d}{d x} \frac{a_{n-1}(x)}{a_{n}(x)}\right. \\
& \left.-\frac{(n-2)^{2} a_{1}(x) a_{n-1}^{2}(x)}{n(n-1) a_{n}^{3}(x)}\right)
\end{aligned}
$$

where $V_{0}(x)$ is given in Lemma 2.5 .

Proof. We compute in (2.2) with $s=n$ the coefficients of $y$ and we have

$$
V_{0}^{\prime}(x)+2 V_{2}(x) a_{0}(x)-2 V_{0}(x) a_{2}(x)=0 .
$$

Using Lemmas 2.3 and 2.5 we get

$$
\begin{gathered}
\frac{d V_{0}(x)}{d x}+\frac{2 a_{2}(x) a_{0}(x)}{a_{n}(x)}-2 a_{2}(x)\left(\frac{a_{0}(x)}{a_{n}(x)}+\frac{1}{n a_{n}(x)} \frac{d}{d x} \frac{a_{n-2}(x)}{a_{n}(x)}\right. \\
\left.-\frac{(n-2)}{n(n-1)} \frac{a_{n-1}(x)}{a_{n}(x)^{2}} \frac{d}{d x} \frac{a_{n-1}(x)}{a_{n}(x)}-\frac{(n-2)^{2} a_{1}(x) a_{n-1}^{2}(x)}{n(n-1) a_{n}^{3}(x)}\right) .
\end{gathered}
$$

This concludes the proof of the lemma.

Lemma 4.5. We have

$$
\begin{aligned}
& \frac{a_{0}(x)}{(n-1)} \frac{d}{d x} \frac{a_{n-1}(x)}{a_{n}(x)}+\frac{(n-2) a_{0}(x) a_{1}(x) a_{n-1}(x)}{(n-1) a_{n}(x)} \\
& =\frac{a_{1}(x)}{n} \frac{d}{d x} \frac{a_{n-2}(x)}{a_{n}(x)}-\frac{(n-2)}{n(n-1)} \frac{a_{1}(x) a_{n-1}(x)}{a_{n}(x)} \frac{d}{d x} \frac{a_{n-1}(x)}{a_{n}(x)}-\frac{(n-2)^{2} a_{1}^{2}(x) a_{n-1}^{2}(x)}{n(n-1) a_{n}^{2}(x)} .
\end{aligned}
$$


Proof. We compute in (2.2) with $s=n$ the coefficients of $y^{0}$ and we have

$$
a_{0}(x) V_{1}(x)=a_{1}(x) V_{0}(x) .
$$

Using Lemmas 2.4 and 2.5 we get

$$
\begin{aligned}
& \frac{a_{0}(x) a_{1}(x)}{a_{n}(x)}+\frac{a_{0}(x)}{(n-1) a_{n}(x)} \frac{d}{d x} \frac{a_{n-1}(x)}{a_{n}(x)}+\frac{(n-2) a_{0}(x) a_{1}(x) a_{n-1}(x)}{(n-1) a_{n}^{2}(x)} \\
& =\frac{a_{1}(x) a_{0}(x)}{a_{n}(x)}+\frac{a_{1}(x)}{n a_{n}(x)} \frac{d}{d x} \frac{a_{n-2}(x)}{a_{n}(x)}-\frac{(n-2)}{n(n-1)} \frac{a_{1}(x) a_{n-1}(x)}{a_{n}(x)^{2}} \frac{d}{d x} \frac{a_{n-1}(x)}{a_{n}(x)} \\
& -\frac{(n-2)^{2} a_{1}^{2}(x) a_{n-1}^{2}(x)}{n(n-1) a_{n}^{3}(x)} .
\end{aligned}
$$

After simplifying by $1 / a_{n}(x)$, the lemma follows.

Proof of Theorem 1.3. The proof of Theorem 1.3 is an immediate consequence of Lemmas 4.1, 4.2, 4.4 and 4.5 .

\section{Acknowledgements}

The first author is partially supported by a MCYT/FEDER grant MTM2008-03437, a CIRIT grant number 2009SGR-410, an ICREA Academia, and two grants FP7-PEOPLE-2012-IRSES 316338 and 318999, and FEDER/UNAB10-4E-378. The second author has been supported by FCT (grant PTDC/MAT/117106/2010 and through CAMGSD).

\section{References}

[1] E Casas-Alvero, Singularities of Plane Curves, London Math. Soc. Lecture Note Ser., vol. 276, Cambridge University Press, Cambridge, 2000.

[2] J. Chavarriga, H. Giacomini, J. Giné and J. Llibre, Darboux integrability and the inverse integrating factor, J. Differential Equations 194 (2003), 116-139.

[3] E.S. Cheb-Terrab and A.D. Roche, Abel ODE's: equivalence and integrable classes, Comput. Phys. Comm. 130 (2000), 204-231.

[4] E.S. Cheb-Terrab and A.D. Roche, An Abel ordinary differential equation class generalizing known integrable classes, Eur. J. Appl. Math. 14 (2003), 217-229.

[5] J. Écalle, Introduction deux fonctions analysables et preuve constructive de la conjecture de Dulac, Hermann, 1992.

[6] J. Giné and M. Grau, Weierstrass integrability of differential equations, Appl. Math. Lett. 23 (2010), 523-526.

[7] J. Giné and J. Llibre, On the integrable rational Abel differential equations, Z. Angew. Math. Phys. 61 (2010), 33-39.

[8] E. Kamke, Differentialgleichungen "losungsmethoden und losungen", Col. Mathematik und ihre anwendungen vol. 18, Akademische Verlagsgesellschaft Becker und Erler Kom-Ges., Leipzig (1943).

[9] J. Llibre and C. Valls, Generalized Weierstrass integrability of the Abel differential equations, Mediterranean Journal of Mathematics, DOI 10.1007/s00009-013- 0266-0 (2013), pp 12.

[10] J. Llibre and C. Valls, Generalized Weierstrass integrability for the complex differential equations $d y / d x=a(x) y^{4}+b(x) y^{3}+c(x) y^{2}+d(x) y+e(x)$, Appl. Math. Letters 26 (2013), 836-841. 